\title{
Use of non-radioactive detection in SSCP, direct DNA sequencing and $\mathrm{LOH}$ analysis
}

\author{
I Petersen, M B Reichel, M Dietel
}

\begin{abstract}
Aims-To develop a protocol that is applicable to single strand conformation polymorphism (SSCP), direct sequencing and loss of heterozygosity analysis of DNA. Methods-The protocol is based on the detection of biotinylated DNA by a Streptavidin-alkaline phosphatase conjugate. Biotinylation of DNA was achieved by using $5^{\prime}$-end biotinylated primers for PCR. After polyacrylamide gel electrophoresis, the DNA fragments were transferred to a nylon membrane by contact blotting. Depending on the alkaline phosphatase substrate, DNA was visualised either colorimetrically or by chemiluminescence.

Results-The method was verified by the identification and characterisation of $p 53$ mutations by SSCP analysis and direct DNA sequencing, as well as the assessment of DNA loss in human lung carcinomas by microsatellite polymorphism allelotyping. Conclusions-The protocol is simple, does not require specialised equipment and would be particularly useful for laboratories with experience in Streptavidin-biotin methodology.
\end{abstract}

(f Clin Pathol: Mol Pathol 1996;49:M118-M121)

Keywords: SSCP, DNA sequencing, DNA polymorphism analysis.

Molecular genetic analysis will soon be an integral part of tumour diagnosis. Thus, there is an increased need for non-radioactive protocols. Single strand conformation polymorphism (SSCP) analysis and direct DNA sequencing are used for the detection of mutations at the nucleotide level-for example, DNA point mutations, whereas loss of heterozygosity (LOH) analysis, or microsatellite polymorphism allelotyping, defines deletions in the tumour genome at the chromosomal or gene level. We have developed a non-radioactive protocol based on the detection of biotinylated DNA by a Streptavidin-alkaline phosphatase conjugate compatible with all three methods-that is, SSCP analysis, DNA sequencing and $\mathrm{LOH}$ analysis.

\section{Methods}

We have presented the protocol according to the technical steps that SSCP, direct DNA sequencing and microsatellite polymorphism analysis have in common, rather than describing the methods separately.

\section{POLYMERASE CHAIN REACTION}

A single pair of $5^{\prime}$-biotinylated primers was used when SSCP analysis and direct DNA sequencing were applied concomitantly for the identification of DNA mutations. PCR conditions-for example, $\mathrm{MgCl}_{2}$ concentration, hot start, annealing temperature, number of cycles, were optimised to reduce unspecific amplification and were identical for both methods. The sequencing reaction was performed by cycle sequencing according to a modified fmol (Promega) protocol. Briefly, $4 \mu \mathrm{l}$ aliquots of a $17 \mu \mathrm{l}$ sequencing mixture containing $1.5 \mathrm{pmol}$ of one biotinylated primer, either sense or antisense, purified double stranded DNA template (Magic PCR Preps DNA Purification System, Promega), sequencing reaction buffer, and Taq polymerase were added to the four different $\mathrm{d} / \mathrm{ddNTP}$ termination mixtures. Only one of the two primers was $5^{\prime}$ biotinylated for amplifying microsatellite polymorphisms. The reaction volumes were $20 \mu \mathrm{l}$

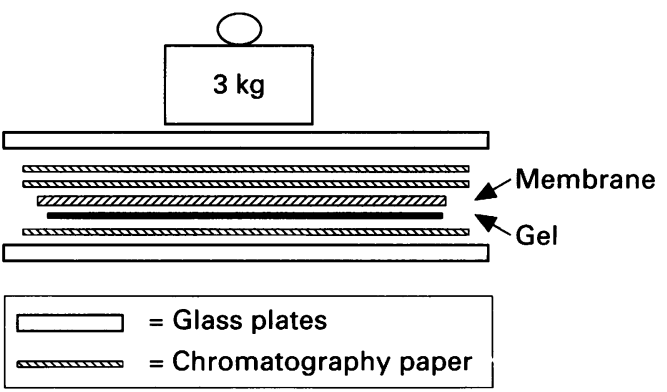

Figure 2 DNA transfer. 
DNA fragments on membrane
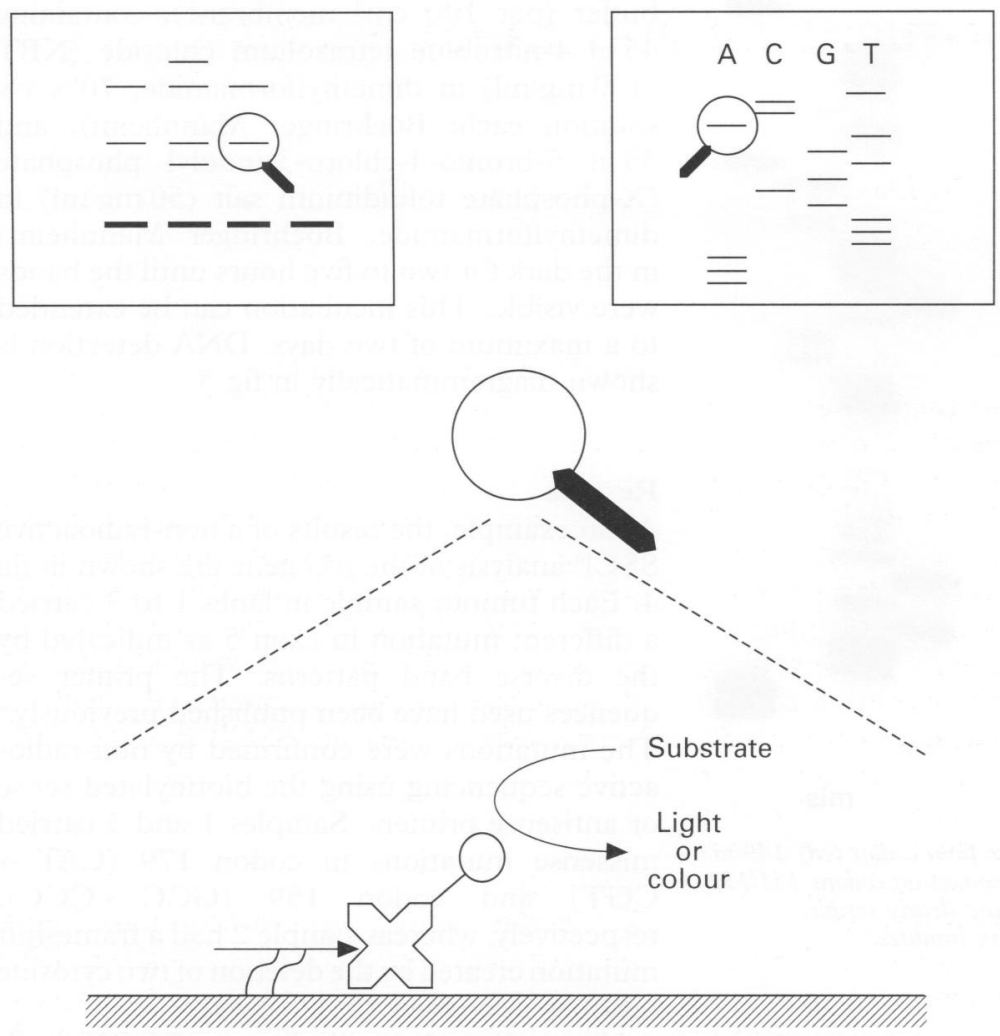

Membrane

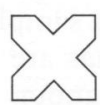

Streptavidin

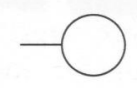

Alkaline phosphatase
5'-Biotinylated DNA

Figure 3 DNA detection. for SSCP and microsatellite polymorphism analysis. Aliquots of these reaction volumes were used for agarose gel electrophoresis and polyacrylamide gel electrophoresis (PAGE).

\section{POLYACRYLAMIDE GEL ELECTROPHORESIS}

PAGE was carried out on a standard sequencing electrophoresis unit (Model STS 45, International Biotechnologies) (fig 1). For SSCP and microsatellite polymorphism analysis, PCR aliquots were added to formamide containing sequencing stop solution, ${ }^{1}$ which accounts for differences in the amount of PCR product produced by each sample. After denaturation by heating at $95^{\circ} \mathrm{C}$ for eight minutes, the samples were loaded onto a $0.4 \mathrm{~mm}$ thick polyacrylamide gel. Non-denaturing PAGE, which reveals the conformational differences among single stranded DNA molecules, was carried out by using polyacrylamide gels with an acrylamide:bisacrylamide ratio that varied between 19:1 and 99:1. Denaturing PAGE in microsatellite polymorphism analysis and DNA sequencing was done with 19:1 acrylamide/ bisacrylamide gels containing $7 \mathrm{M}$ urea. The concentration of the polyacrylamide gels was adjusted according to the length of the fragment to be analysed. ${ }^{1}$

\section{DNA TRANSFER BY CONTACT BLOTTING}

After PAGE, the DNA was transferred to a nylon membrane by contact blotting. One of the glass plates that covered the polyacrylamide gel was removed, and the gel separated from the second glass plate by a dry sheet of chromatography paper (Whatman 3MM). The part of the gel containing no information was cut off and the remaining gel and the chromatography paper were placed on a dry, clean glass plate. The nylon membrane (Nytran NY 13 $\mathrm{N}$, Schleicher and Schuell or Hybond $\mathrm{N}+$, Amersham) cut to the appropriate size was briefly incubated in $1 \times \mathrm{TBE}$ and laid on the gel. Two dry sheets of chromatography paper were placed on top. Care was taken to avoid formation of air bubbles between the gel and the membrane. Finally, this sandwich was covered by a second glass plate and subjected to a $2-3 \mathrm{~kg}$ weight (fig 2). After blotting for 30-60 minutes, the DNA was fixed to the nylon membrane by ultraviolet crosslinking for three minutes. An ultraviolet crosslinker or the ultraviolet light of a cell culture hood or an ultraviolet transilluminator was used.

DETECTION OF MEMBRANE BOUND DNA

The membrane was transferred to a glass bath and washed twice for 15 minutes each in blocking buffer $(0 \cdot 2 \%$ I-block reagent, Tropix, or $0 \cdot 2 \%$ casein (Sigma) in $0.5 \%$ SDS (sodium dodecylsulphate), $1 \times$ phosphate buffered saline (PBS)). This was followed by incubation for 20 minutes with a Streptavidin-alkaline phosphatase complex (AVIDx-AP conjugate,
Figure 4 Non-radioactive SSCP analysis of exon 5 of the $\mathrm{p} 53$ gene. Each sample in lanes 1-3(from left to right) carried a different mutation. Visualisation was by colorimetry. 


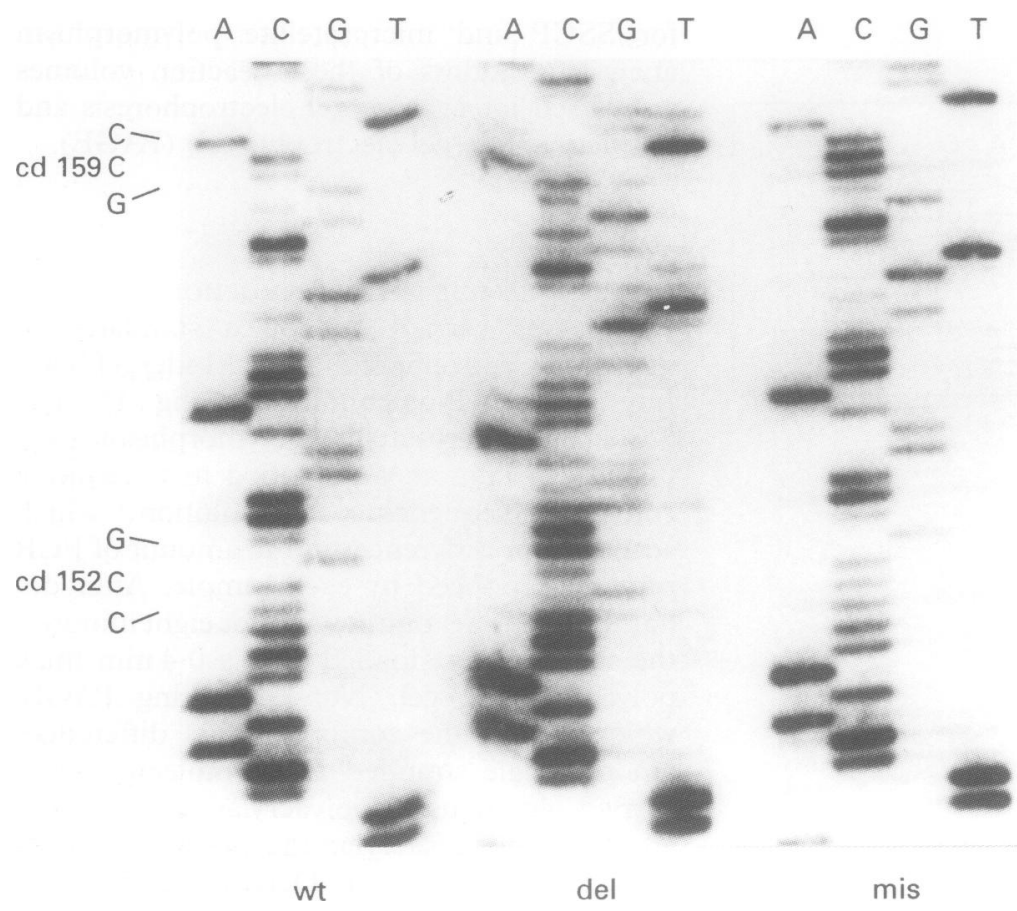

Figure 5 Non-radioactive DNA sequencing showing the sequence from codon (cd) 149 to codon 161 of the $\mathrm{p} 53$ gene. The deletion (del) of two cytosine residues on codons 151/152 and the missense (mis) mutation in codon 159 (GCC $\rightarrow C C C$ ) are clearly visible.

Detection was by chemiluminescence with an exposure time of five minutes. ray film cassette. For colorimetric detection, the membrane was incubated in $10 \mathrm{ml}$ DEA buffer (per $100 \mathrm{~cm}^{2}$ membrane), containing $45 \mu \mathrm{l}$ 4-nitroblue tetrazolium chloride (NBT $(100 \mathrm{mg} / \mathrm{ml})$ in dimethylformamide, $70 \% \mathrm{v} / \mathrm{v}$ solution each; Boehringer Mannheim), and $35 \mu \mathrm{l}$ 5-bromo-4-chloro-3-indolyl phosphate (X-phosphate toluidinium salt $(50 \mathrm{mg} / \mathrm{ml})$ in dimethylformamide; Boehringer Mannheim) in the dark for two to five hours until the bands were visible. This incubation can be extended to a maximum of two days. DNA detection is shown diagrammatically in fig 3 .

\section{Results}

As an example, the results of a non-radioactive SSCP analysis of the $p 53$ gene are shown in fig 4. Each tumour sample in lanes 1 to 3 carried a different mutation in exon 5 as indicated by the diverse band patterns. The primer sequences used have been published previously. ${ }^{2}$ The mutations were confirmed by non-radioactive sequencing using the biotinylated sense or antisense primers. Samples 1 and 3 carried missense mutations in codon 179 (CAT $\rightarrow$ CGT) and codon 159 (GCC $\rightarrow$ CCC), respectively, whereas sample 2 had a frameshift mutation created by the deletion of two cytosine residues in codons $151 / 2$ (fig 5). ${ }^{3}$

Non-radioactive assessment of $\mathrm{LOH}$ is illustrated in fig 6. A dinucleotide repeat polymorphism located on chromosome $3 p$ at the human thyroid receptor $\beta$ gene was analysed. ${ }^{4}$ Paired samples of four patients with lung carcinomas are shown. $\mathrm{LOH}$ is clearly visible in the tumour samples of patients 2 and 4 as indicated by the loss of one band in lanes 3 and 7 (from left to right), respectively. Patient 1 was homozygous - that is, only one allele is visible, whereas the tumour of patient 3 retained heterozygosity. Note that four alleles of this microsatellite polymorphism are represented in fig 6 . One of the primers used to amplify the 197-209 base pair repeat was biotinylated at the $5^{\prime}$-end.

We observed no major differences in sensitivity when DNA was detected by chemiluminescence or colorimetry. Biotinylation of DNA at the $5^{\prime}$-end did not significantly alter the migration properties during PAGE in SSCP analysis, which was assessed by comparing the band pattern of radioactive SSCP analysis with non-biotinylated primers to the banding pattern of the non-radioactive method (fig 7).

\section{Discussion}

As for haematopoietic tumours, the characterisation of the genetic alterations in solid neoplasms will extend and refin' morphological and immunohistochemical tumour diagnosis, as cancer genetics can reveal specific events underlying tumour initiation and progression. ${ }^{25}$ The panel of basic techniques for molecular genetic tumour analysis includes SSCP, direct DNA sequencing and $\mathrm{LOH}$ analysis. Although non-radioactive protocols exist, few of them can be applied for all three methods without the need for large and expensive equipment. lution was removed by opening and resealing the plastic bag at one edge. Finally, the membrane was exposed to $x$ ray film (XAR 5, Kodak) for up to 30 minutes in a light proof $x$ 


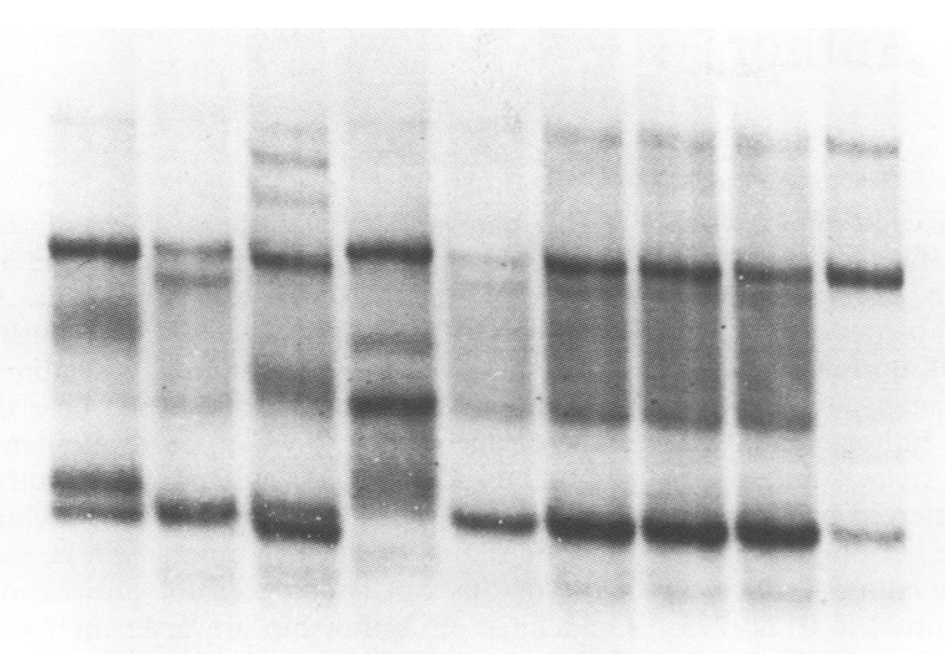

A

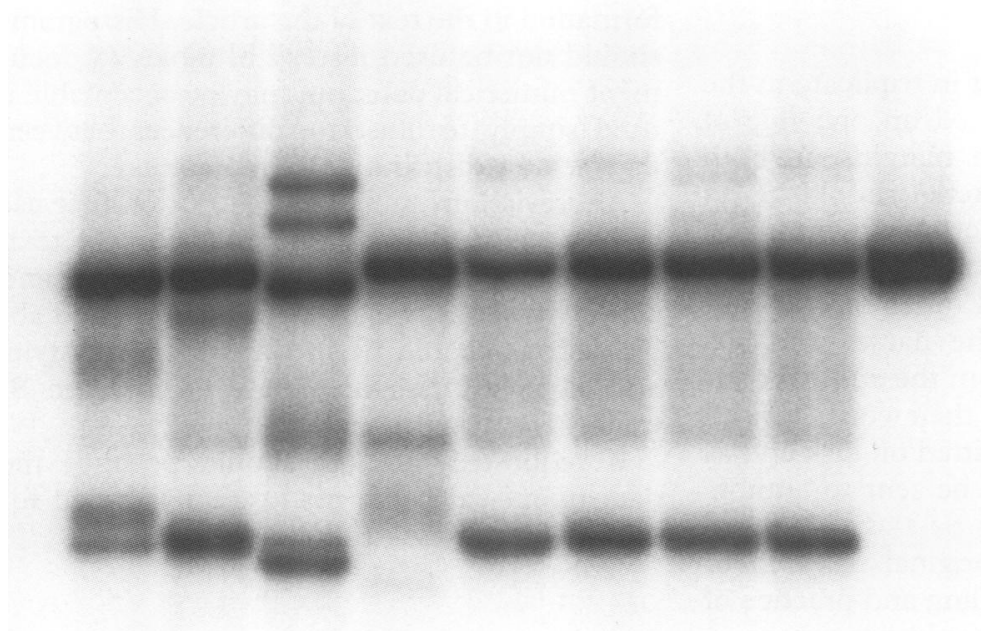

B

Figure 7 Results of a non-radioactive ( $A$ ) and radioactive SSCP analysis (B) of samples with different mutations of the $\mathrm{p} 53$ gene showing a similar banding pattern.

Ethidium bromide ${ }^{6}$ or silver staining ${ }^{7}$ can be used to visualise double and single stranded DNA and are thus primarily used in SSCP analysis. Selective visualisation of a single DNA strand of a PCR product is particularly important in $\mathrm{LOH}$ analysis as typing of alleles by dinucleotide repeats might be obscured by shadow bands. ${ }^{8}$ This is achieved by hapten labelling at the $5^{\prime}$-end of either one or both PCR primers. As biotin is robust and widely used it was chosen as the hapten for $5^{\prime}$-end labelling.

The protocol presented here was devised to take advantage of the similarities among the three methods based on PCR and PAGE. As SSCP analysis and direct DNA sequencing are frequently used together in the detection of DNA mutations, the protocol was set up such that a single pair of primers and similar PCR conditions can be used in both methods. PAGE can be carried out on the same electrophoresis unit in all three methods. Visualisation is possible either by chemiluminescence or colorimetry giving the protocol great flexibility. We regard the procedure described herein as reliable and thus helpful for the introduction of non-radioactive molecular genetic methods to pathology.

The technical assistance of Gesine Heinemann and Martina Bimmler is gratefully acknowledged. We also thank Andreas von Deimling for inspiring discussions on molecular genetic analysis of solid tumours by DNA polymorphisms.

1 Sambrook J, Fritsch EF, Maniatis T. Molecular cloning: a laboratory manual. Cold Spring Harbor: Cold Spring Harbor Laboratory Press, 1989.

2 Petersen I, Ohgaki H, Ludeke BI, Kleihues P. p53 mutations in phenacetin-associated urothelial carcinomas. Car cinogenesis 1993;14:2119-22.

3 Reichel MB, Ohgaki H, Petersen I, Kleihues P. p53 mutations in primary human lung tumours and their metastases. $\mathrm{Mol}$ Carcinog 1994;9:105-9.

4 Sakurai A, Bell GI, DeGroot LJ. Dinucleotide repeat polymorphism in the human thyroid hormone receptor $\beta$ gene morphism in the human thyroid hormone receptor $\beta$ gene
(THRB) on chromosome 3. Nucleic Acids Res 1992;19: 6661 .

5 Rodriguez E, Sreekantaiah C, Chaganti RSK. Genetic changes in epithelial solid neoplasia. Cancer Res 1994;54: 3398-406.

6 Mohabeer AJ, Hiti AL, Martin WJ. Non-radioactive single strand conformation polymorphism (SSCP) using the Pharmacia 'PhastSystem'. Nucleic Acids Res 1991;19:3154. 7 Yap EPH, McGee JO. Nonisotopic SSCP and competitive Nucleic Acids Res 1992;20:145.

$8 \mathrm{Litt} \mathrm{M}$, Hauge X, Sharma V. Shadow bands seen when typing polymorphic dinucleotide repeats: some causes and cures. Biotechniques 1993;15:280-4. 